\title{
Effect of cyclodextrin encapsulation on the photocyclization of diphenylamine : Cavity imposed restriction on the reaction rate
}

\author{
Debjani Sur ${ }^{\mathrm{a}}$, Pradipta Purkayastha ${ }^{\mathrm{a}}$, Subhash Chandra Bera ${ }^{\mathrm{a}}$ and Nitin Chattopadhyay ${ }^{\mathrm{a}, \mathbf{b}_{*}}$ \\ "Department of Chemistry, Jadavpur University, Calcutta - 700 032, India \\ ${ }^{b}$ Department of Chemistry, Coimbra University, Coimbra - 3049, Portugal
}

Received 02 August 2000: accepted 10 October 2000

\begin{abstract}
The kinetics of the photocyclization of diphenylamine (DPA) to carbazole (CAZL) has been studied fluorometrically in air-equilibrated aqueous solution as well as in constrained microheterogeneous media provided by $\alpha-, \beta$-, and $\gamma$-cyclodextrins (CDs). It is observed that the fluorophore is embedded within the $\mathrm{CD}$ cavities without any alteration of the overall reaction quantum yield in the different environments. However, the rate of the photoreaction is modified remarkably within the $C D$ environments. A restriction on the intramolecular rotation of the phenyl planes of DPA, imposed by the steric rigidity within the $C D$ cavities, has been ascribed to be responsible for the suppression of the reaction rates within the CD environments. A semi-empirical (AM1) calculation gives the molecular dimension of the substrate and corroborates the proposition from a consideration of the cavity size of the different cyclodextrins.

(C) 2000 Elsevier Science B.V. All rights reserved.
\end{abstract}

* Corresponding author; pcnitin@yahoo.com. 


\section{INTRODUCTION}

The intramolecular photocyclization of diphenylamine (DPA) and its derivatives to yield carbazole (CAZL) and its derivatives has been the subject of extensive research in a number of laboratories since long for the complex and interesting features of the reaction [1-14]. The photoconversion takes place in degassed or air-equilibrated solutions. The problem was initiated by Parker and Barnes [1]. Bowen and Eland, in an early paper [2], reported that the photocyclization of DPA involves a different state from the original excited state and further used mass-spectrometry to find that the amount of molecular hydrogen produced is comparable to that of CAZL formed. Hlash-excitation study made by Grellmann et al. $[3,4]$ showed that the photoconversion mechanism involves the formation of a triplet transient that is converted to another intermediate absorbing at 610 $\mathrm{nm}$, which finally gives CAZL. The final intermediate has been said to have a closed ring structure which may either revert back to give DPA or else can undergo CAZL formation [3]. The mechanism of the multi-step reaction has been proposed by Rahn et al. [9] which is supported by the works in different laboratories [10,11]. The general scheme for the reaction can be summarized as Scheme 1 .

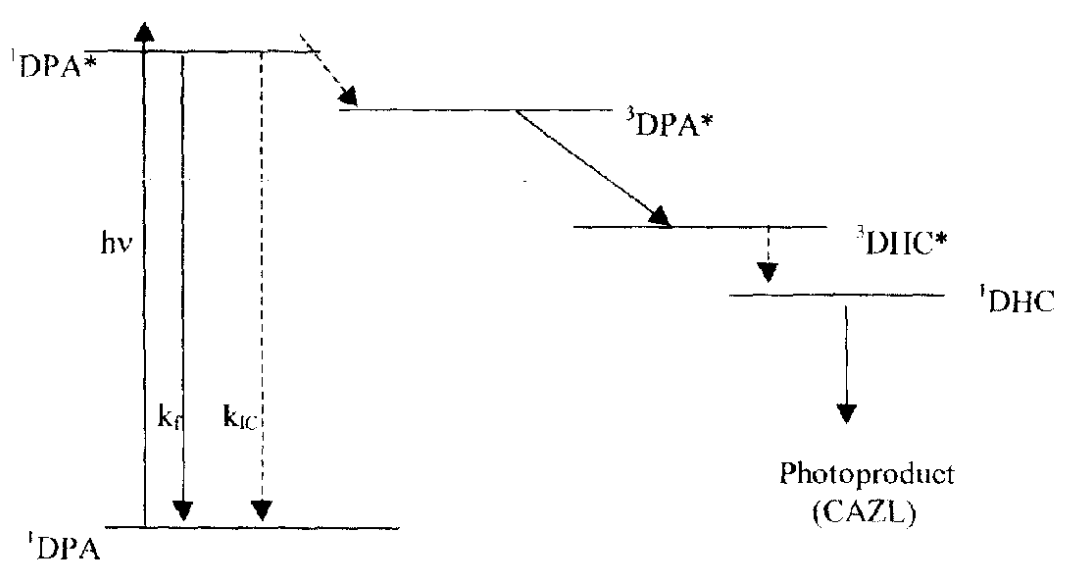

Scheme 1 : Multistep photoconversion of DPA into CAZL. 
Where DPA, DHC and CAZL are the diphenylamine, dihydrocarbazole (intermediate) and carbazole in the corresponding ground states, ${ }^{*}$ in the right hand superscript indicates the species in the photoexcited state and 1 and 3 in the left hand superscript corresponds to the species in the singlet and triplet states respectively. Suzuki et al. [10] have studied the energetics of the photocylization of DPA in methanol (MeOH) solution by time-resolved thermal lensing (TRTL) technique. Considering the fact that DPA differs from the other two members of the series, viz., N-methyldiphenylamine (MDPA) and triphenylamine (TPA), Chattopadhyay et al. have recently studied the energetics of the photoreaction of TPA in alkane as well as alcoholic solvents using the time-resolved photoacoustic calorimetric technique [11]. In the same work, they have also assigned the long lived intermediate as a zwitterionic species for the first time. All the studies relating to the above mentioned photocyclization reaction have, however, been performed only in homogeneous environments. In a recent report, we endeavored to initiate the study of this reaction in microhetergeneous environment [14].

As is known, cyclodextrins (CDs) are interesting microvessels capable of embedding appropriately sized fluorophores. The photophysics and the photochemistry of the embedded fluorophore are modified a lot because of the difference of the microenvironment from that of the bulk [14-25]. Cyclodextrins influence the photoprocesses by two possible ways: providing a reduced polarity as well as an enhanced steric rigidity within the CD core.

The present work deviates from the mechanistic aspect of the photoconversion reaction of DPA. Here we have studied the kinetics of the photocyclization reaction of DPA in aqueous and aqueous CD solutions using the fluorometric technique. Our intention lies in studying the effect of encapsulation of the reactant into different $\mathrm{CD}$ cavities on the reaction kinetics. This is particularly important for this type of reaction as it involves a single fluorophore molecule for the reaction. The different cavity size in $\alpha-\beta-$ and $\gamma-C D$ has been found to affect the rate of the photoreaction differently. 


\section{EXPERIMENTAL}

Diphenylamine and carbazole (both from Aldrich) were purified following the procedures as mentioned elsewhere $[26,27]$. Good quality of cyclodextrins $(\alpha-\beta$ - and $\gamma$-) were procured from Fluka and were used as received without further purification. Triple distilled water was used for the preparation of the experimental solutions. All the experiments were performed with air cquilibrated solutions. The preparations of the solutions were done under deemed light so as to avoid any photochemical transformation beforehand. The method of preparation of DPA and CAZL solutions of known concentration has been described in our previous work [14].

Shimadzu MPS 2000 spectrophotometer and Spex Fluorolog 2 spectrofluorimeter were used for the absorption and fluorescence measurements. For the geometry optimization of DP $\Lambda$, we have used the semiempirical $\Lambda M 1$ method supported by the Hyperchem 5.01 package from Hypercube Inc., Canada. Utility of the AM1 method in getting reliable structural data has already been established $[11,28-31]$.

\section{RESULTS AND DISCUSSION}

As mentioned in the earlier paper [14], the absorption spectrum of the aqueous solution of DPA is unstructured while that of CAZI is distinctly a structured one. The former had a maximum at $\sim 280 \mathrm{~nm}\left(\varepsilon=20,600 \mathrm{M}^{-1} \mathrm{~cm}^{-1}\right)$ and the latter at $\sim 292 \mathrm{~nm}\left(\varepsilon=16,000 \mathrm{M}^{-1}\right.$ $\mathrm{cm}^{-1}$ ). The excitation wavelength, for the present experiment, was set to $292 \mathrm{~nm}$, as the molar extinction coefficients of the two molecular systems are nearly the same at this wavelength [14]. The absorption spectrum of DPA showed no appreciable change on addition of the CDs to the solution. 
$-358 \mathrm{~nm}$. In aqueous solutions the fluorescence quantum yields of DPA and CAZL were estimated to be 0.06 and 0.21 respectively against quinine sulfate solution in $0.1 \mathrm{~N} \mathrm{H}_{2} \mathrm{SO}_{4}$ $(\phi=0.54)$ [32]. It is important to point out here that the portion of the DPA solution used for the absorption study must not be used for the emission studies as an appreciable portion of the photochemical reaction takes place during the other study. So, a fresh part from the stock solution, kept in dark, must be used. Contrary to the absorption spectrum, the emission spectrum of DPA changes with the addition of CD to the aqueous solution. With an increase in the $\alpha-C D$ concentration there was hardly any change in the fluorescence yield of DPA recorded at zero time. But a hypsochromic shift was observed reflecting that the microenvironment around the probe was different from the bulk aqueous phase. With increased concentrations of $\beta$ - and $\gamma$-CD, DPA shows a gradual increase (although small) in the fluorescence yield with a clear hypsochromic shift. There is also a shortening of the full width half maximum (FWHM) for the emission band with increased $\beta-C D$ concentration. This, however, remains nearly constant in the other two CDs. The fluorescence study, thus, indicates an inclusion of the fluorophore within the CDs. It further reflects that the interaction of the fluorophore is maximum with $\beta-C D$ and minimum with $\alpha-C D$. A comparative study of the emission maximum (EM) and FWHM for the fluorescence of DPA in aqueous $(E M=390 \mathrm{~nm}, F W H M=88 \mathrm{~nm})$ and aqueous $\beta$ $\mathrm{CD}$ solution $(\mathrm{EM}=368 \mathrm{~nm}, \mathrm{FWHM}=78 \mathrm{~nm})$ with those in homogeneous solvents differing in polarity (the above two parameters for DPA in methanol, tetrahydrofuran and n-heptane being $357 \mathrm{~nm}$ and $59 \mathrm{~nm} ; 349 \mathrm{~nm}$ and $45 \mathrm{~nm} ; 334 \mathrm{~nm}$ and $41 \mathrm{~nm}$ respectively) also indicates that the fluorophore experiences a less polar environment within the $\beta$-CD core. Fig. 1 shows the fluorescence spectra of DPA at zero time in the presence of different cyclodextrins. Presence of isoemissive points (at $379 \mathrm{~nm}, 388 \mathrm{~nm}$ and $387 \mathrm{~nm}$ for $\alpha-, \beta$ - and $\gamma$-CD respectively) in the fluorescence spectra demonstrates the formation of $1: 1$ inclusion complex between the fluorophore and the CD systems. The association constants of DPA with $\beta$ - and $\gamma$-CD have been determined from the fluorescence study following the double reciprocal plot. The detail of the technique has been described elsewhere [33]. The double reciprocal plot was linear substantiating the one-to-one complexation between DPA and 

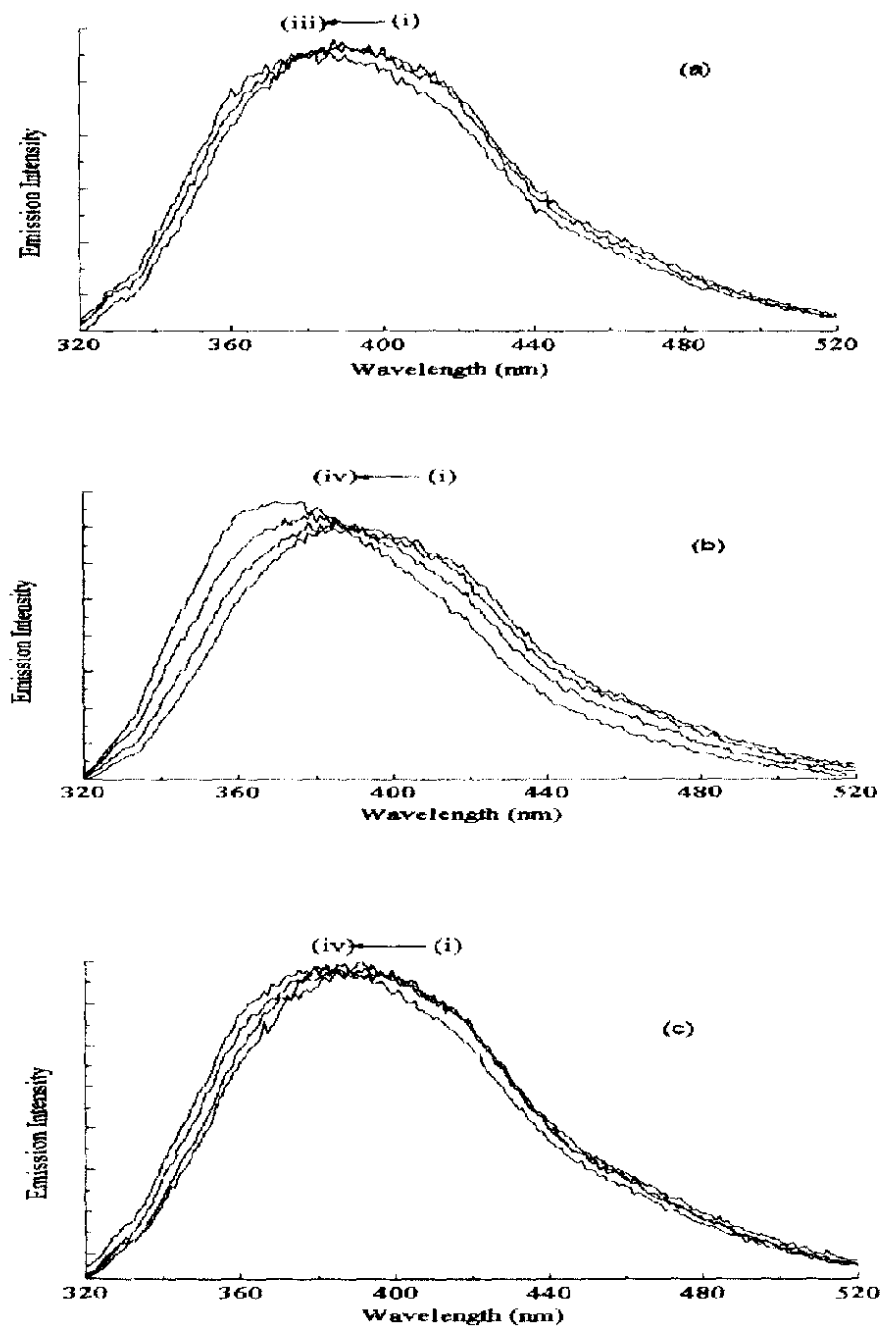

Fig. 1: Fluorescence spectra of DPA (at zero time) in aqueous (a) $\alpha-\mathrm{CD}$ (b) $\beta-\mathrm{CD}$ and (c) $\gamma$-CD solutions. In (a), (i) to (iii) refer to $\alpha$-CD concentrations 0.6 .75 and 15 $\mathrm{mM}$ respectively; in (b), (i) to (iv) refer to $\beta-C D$ concentrations of $0,0.25,0.75$ and $1.5 \mathrm{mM}$ respectively and in (c). (i) to (iv) refer to $\gamma-C D$ concentrations of 0 , $2.1,3.0$ and $6.0 \mathrm{mM}$ respectively. Concentration of DPA is $1.5 \times 10^{-6} \mathrm{M}$ in all the cases. 
cyclodextrin. The values of the association constants, thus determined were 135 and 62 $\mathrm{mol}^{-1} \mathrm{dm}^{3}$ for DPA $-\beta-\mathrm{CD}$ and DPA $-\gamma$-CD systems respectively. The method, however, could not be applied to the DPA - $\alpha-\mathrm{CD}$ system, as the increase in the fluorescence with the addition of $\alpha-C D$ was inappreciable.

On keeping the aqueous solution of DPA, exposed to $292 \mathrm{~nm}$ radiation, the fluorescence spectrum changes with time. The unstructured DPA fluorescence is gradually transformed into the structured fluorescence of CAZL. This is associated with a movement of the peak maximum from $390 \mathrm{~nm}$ to $358 \mathrm{~nm}$ as well as an increase in the fluorescence yield (because of the higher fluorescence quantum yield of CAZL compared to that of DPA (vide infra). Thus, monitoring the total fluorescence yield provides a convenient way to study the course of the photoconversion reaction of DPA to CAZI. Fig. 2 represents the plots of the total fluorescence upon excitation of DPA in aqueous and aqueous CD solutions with

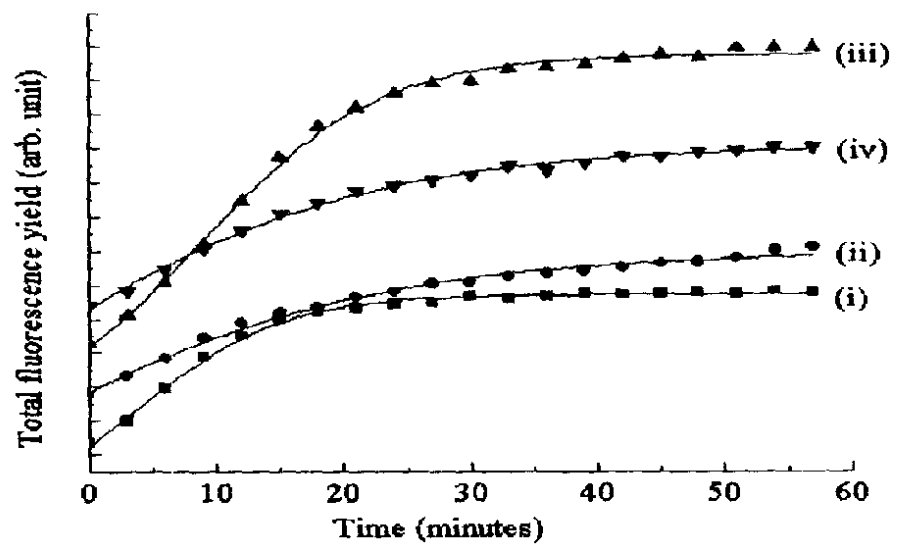

Fig. 2: Plot of total fluorescence upon excitation of DPA vs. time in (i) aqueous, (ii) $2.25 \mathrm{mM} \alpha-\mathrm{CD}$, (iii) $1.5 \mathrm{mM} \quad \beta-\mathrm{CD}$ and (iv) $1.05 \mathrm{mM} \gamma-\mathrm{CD}$ solutions (concentration of DPA is $1.5 \times 10^{-6} \mathrm{M}$ ). 
time. It is evident from the figure that the photoconversion reaction is quite slow (time range in minutes). It is important to notice that the time required for leveling of the fluorescence is greater for the CD solutions, in general, compared to that in pure aqueous solution reflecting that the reaction is slower for the embedded fluorophore compared to the unbounded one. The time required for leveling is experimentally found to be in the order : water $<\beta-\mathrm{CD}<\gamma-\mathrm{CD}<\alpha-\mathrm{CD}$. It is pertinent to mention here that in cyclodextrin solutions. all the DPA molecules are supposed to be complexed with CDs as the concentration of the latter is three orders of magnitude higher than that of DPA. To follow the kinetics of the photoconversion of DPA to $\mathrm{CAZL}$ in aqueous and aqueous $\mathrm{CD}$ solutions and to determine the corresponding rate constants we adopt the following method [14].

Initially, at $t=0$, the total fluorescence corresponds to the total amount of DPA present in the solution. With the passage of time (under continuous irradiation), DPA concentration decreases with the forrnation of an equimolar amount of CAZL. Mathematically, this can be represented as :

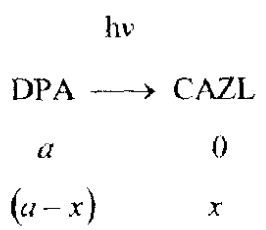

where, $x$ is the number of moles of DPA reacted or moles of CAZL formed, $a$, the initial concentration of DPA and $t$, the time. Considering first order reaction (final plots validate this proposition) onc can write $x=a\left(1-e^{k t}\right), k$ being the first order reaction rate constant. Thus, for dilute solutions of DPA having low optical densities, at any instant of time after the photoreaction starts, the respective concentrations of DPA and CADL becomes $a e^{-k t}$ and $a\left(1-e^{-k t}\right)$. For the present reaction, both the reactant (DPA) and the photoproduct (CAZL) are excited by the exciting radiation at $292 \mathrm{~nm}$ and they emit independently depending on their instantaneous concentrations and fluorescence quantum vields. The total fluorescence at any instant of time $t$ can be correlated with the fractional light absorbed by the components [34] as, 


$$
\phi_{1}=\phi_{C A Z I} I_{C A Z L}+\phi_{D P A} I_{D P A}
$$

$\phi_{l}, \phi_{(A, A],}$ and $\phi_{D P P^{\prime}}$ being the fluorescence quantum yields of the reaction mixture, CAZL and DPA respectively. $I$ 's are the fractional lights absorbed by the species represented by the subscript. Again, $I=I_{0}\left(1-e^{-2.303 \approx l}\right), I_{0}$ being the incident light intensity, $\varepsilon$, the molar extinction coefficient; $c$, the concentration and $l$, the optical path length (here $1 \mathrm{~cm}$ ). Assuming the molar extinction coefficients of DPA and CAZL to be equal (vide infra) and incorporating the concentration terms from the kinetic scheme we arrive at the following final expression:

$$
\log \left[\phi_{C A Z I .}-\frac{\phi_{1}}{2.303 I_{0} \text { laE }}\right]=-\frac{k t}{2.303}+\log \left(\phi_{C A Z I .}-\phi_{D P A}\right)
$$

The term on the left side, when plotted against time, $t$, results straight lines (fig. 3) for

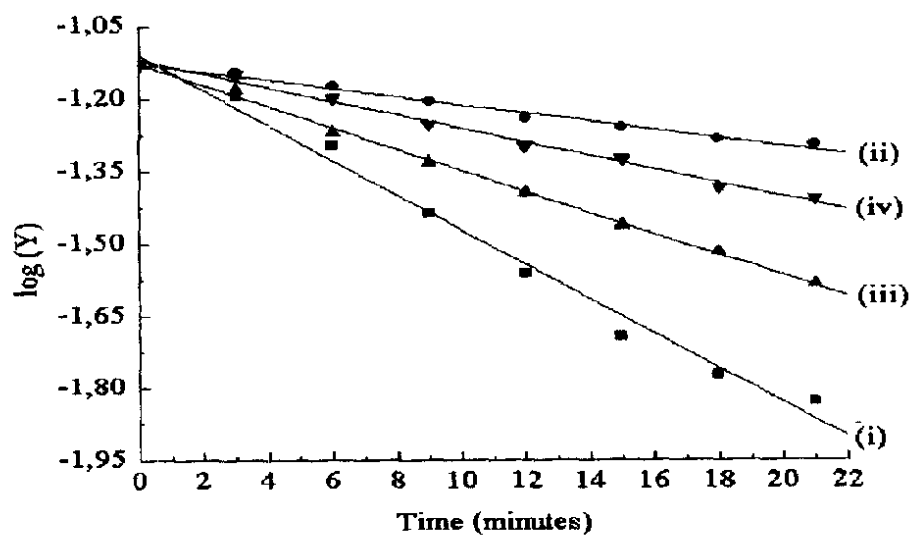

Fig. 3: Plot of $\log (\mathrm{Y})$ vs. time in (i) aqueous, (ii) $2.25 \mathrm{mM} \alpha \mathrm{CD}$, (iii) $1.5 \mathrm{mM} \beta C D$ and (iv) $1.05 \mathrm{mM} \gamma \mathrm{CD}$ solutions. ( $Y=\left[\phi_{C A Z L}-\frac{\phi_{t}}{2.303 I_{0} l a \varepsilon}\right]$, for detail see text). 
aqueous as well as aqueous CD solutions, justifying the 1 st order nature of the reaction in all the environments studied. The slopes of the straight lines give the rate constants of the photocyclization reaction in the respective medium. The estimated rate constants of the reaction in homogencous aqueous and different microheterogencous cyclodextrin environments have beerl tabulated in Table 1.

Table 1: Values of the rate constant, $k$, for the photoconversion reaction of DPA in different environments.

\begin{tabular}{|cc|}
\hline Ervironment & Rate constant (in $\left.\mathrm{min}^{-1}\right)$ \\
\hline Aqueous & $8.3 \times 10^{-2}$ \\
Aqueous $\alpha-\mathrm{CD}$ & $2.2 \times 10^{-2}$ \\
Aqueous $\beta-\mathrm{CD}$ & $5.0 \times 10^{-2}$ \\
Aqueous $\gamma$-CD & $3.2 \times 10^{-2}$ \\
\hline
\end{tabular}

Using the same equation at long times of irradiation of the DPA (after the attainment of the leveling of fluorescence in fig. 2) and adopting the derived rate constant values in different $C D$ environments, one can determine the quantum yield of photoreaction in all the environments. For the aqueous as well as all the CD cnvironments, we determined the quantum yield of reaction to be 0.11 ; consistent with the value reported by Bowen and Eland in homogeneous environment [2]. It is interesting to note that the quantum yield of photoreaction does not depend on the CD concentration.

The estimated rate constants for the photocyclization of DPA in aqueous and various aqueous $\mathrm{CD}$ solutions are thus in the order: water $>\beta-\mathrm{CD}>\gamma-\mathrm{CD}>\alpha-\mathrm{CD}$. This variation in the reaction rate in different environments can be rationalized from a proposition of different modes of enciapsulation of the fluorophore in different CDs which can be realized from a comparison of the geometry of the fluorophore and dimensions of the different $\mathrm{CD}$ 
cavities. We have optimized the geometry of the fluorophore using the semi-empirical AM1 method. The ground state optimized structure reveals that the molecule is nonplanar and the two benzene planes are at an angle of about $52^{\circ}$ with each other (fig. 4). It is, however, now established that the photocyclization reaction proceeds through the formation of a closed ring intermediate, dihydrocarbazole (DHC) [9-11]. In order to form this intermediate the fluorophore molecule must attain a planar (or nearly planar) geometry for which an intramolecular rotation of the molecule is essential. This intramolecular rotation of the embedded fluorophore within the cyclodextrin environments is restricted because of the steric rigidity within the $C D$ cavities. This leads to a lowering of the rate of the photoreaction of DPA within the CD environments compared to that in the aqueous solution. The cavity diameter of $\alpha-, \beta$ - and $\gamma \mathrm{CD}$ are known to be around $4.5 \AA, 7.0 \AA$ and $8.5 \AA$ respectively $[35]$. The varying cavity diameter of different cyclodextrins leads to the inclusion of the probe, DPA, in different ways. $\alpha C D$, because of its small size, is capable

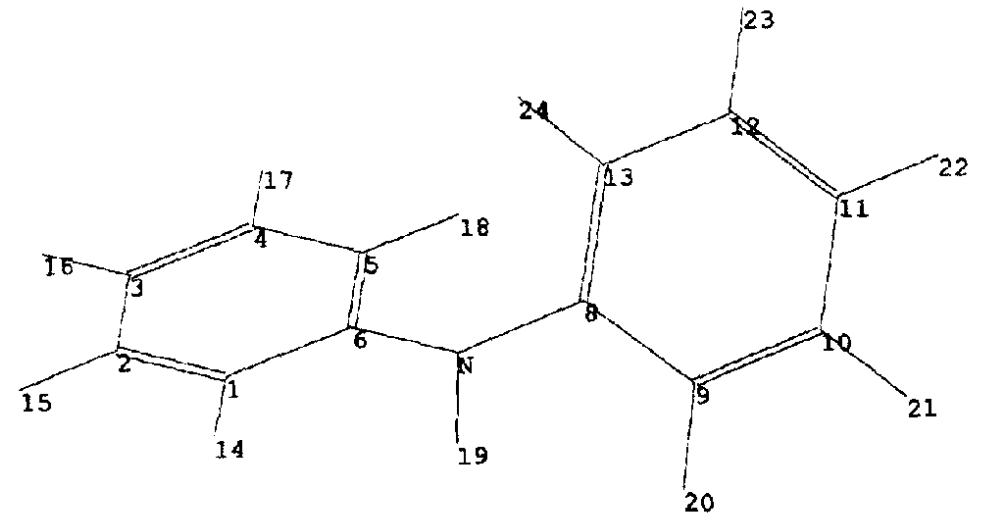

Fig. 4: Optimized geometry of DPA.

of encapsulating only one phenyl ring of the fluorophore $\left(d_{16-18}\right.$ or $\left.d_{15-17} \approx 4.3 \AA\right)$. Thus, while encapsulating one phenyl ring, the $\mathrm{CD}$ wall, probably, acts as a shielding surface between the two reacting centers $\left(C_{5}\right.$ and $C_{13}$ in fig.4), making the cyclization process slowest among all the environments. This shielding effect, however, does not exist in the 
case of $\beta$ - and $\gamma-C D$, because of their larger dimensions. Both of them can accommodate the two carbon centers responsible for the cyclization process. From a comparison of the cavity dimensions of these two CDs and the distances between different atomic centers of DPA, it appears that $\beta-C D$ accommodates the two reacting centers keeping one phenyl ring inside the cavity and the other mostly outside $\left(d_{16-13}\right.$ or $\left.d_{15-13} \approx 6.3 A\right) . \gamma-C D$, however, encompasses almost both the phenyl rings inside the cavity $\left(\mathrm{d}_{15-23} \approx 8.3 \AA\right.$ ). This leads to a difference in the relative rates of cyclization reaction in these two environments. The intramolecular rotation of one phenyl ring of DPA relative to the other is more feasible in $\beta-\mathrm{CD}$ than in $\gamma-\mathrm{CD}$ leading to a faster reaction rate in the former compared to that in the latter. In any case, in both the cases, the intramolecular rotation is restricted compared to when the fluorophore is in pure aqueous solution. Thus, in both $\beta$ - and $\gamma-C D$ environments, the reaction rate is slower than that in the aqueous phase. Since no reactant molecule is permanently blocked for the reaction, inclusion of the fluorophore with the CDs has no effect on the final quantum yield of the photochemical reaction.

\section{CONCLUSION}

The present compreherisive study of the photocyclization of DPA in aqueous and different aqueous cyclodextrin environments leads to the following points:

(i) Interaction of DPA and CDs results in the formation of $1: 1$ inclusion complexes.

(ii) The rate of the cyclization reaction is affected within the microheterogeneous environments. In different CD solutions, the fluorophore is embedded differently depending on the cyclodextrin cavity size; consequently, the reaction rate varies in different environments.

(iii) A remarkable lowering in the rate of the reaction in the CD environments indicates that the photocyclization reaction proceeds through an intramolecular rotation.

(iv) The quantum yield of the photochemical conversion of DPA to CAZL is not modified in the $\mathrm{CD}$ environments. 


\section{ACKNOWLEDGEMENTS}

Financial assistance from the Department of Science and Technology and Council of Scientific and Industrial Research, Govt. of India, is gratefully acknowledged.

\section{REFERENCES}

1 C. A. Parker and W. J. Barnes, Analyst, 82 (1957) 606.

2 E. J. Bowen and J. H. D. Eland, Proc. Chem. Soc. London, (1963) 202.

3 K. H. Grellmann, G. M. Sherman and H. Linschitz, J. Am. Chem. Soc., 85 (1963) 1881.

4 H. Linschitz and K. H. Grellmann, J. Am. Chem. Soc., 86 (1964) 303.

5 G. Bartelt, A. Eychrnueller and K. H. Grellmann, Chem. Phys. Letts., 118 (1985) 568 .

6 U. Kensy, M. M. Gonzalez and K. H. Grellmann, Chem. Phys., 170 (1993) 381.

7 G. C. Terry, V. E. Uffindell and F. W. Willets, Nature, 223 (1969) 1050.

8 E. W. Förster, K. H. Grellmann and H. Linschitz, J. Am. Chem. Soc, 95 (1973) 3108.

9 R. Rahn, J. Schroeder, J. Troe and K. H. Grellmann, J. Phys. Chem, 93 (1989) 7841.

10 T. Suzuki, Y. Kajii, K. Shibuya and K. Obi, Bull. Chem. Soc. Jpn, 65 (1992) 1084.

11 N. Chattopadhyay, C. S. Soares, P. Purkayastha, L. G. Arnaut and S. J. Formosinho, Phys. Chem. Chem. Phys. (in press).

12 E. W. Förster and K. H. Grellmann, Chem. Phys. Letts, 14 (1972) 536.

13 K. H. Grellmann, W. Kuhnle, H. Weller and T. Wolff, J. Am. Chem. Soc., 103 (1981) 6889.

14 D. Sur, P. Purkayastha and N. Chattopadhyay, J. Photochem. Photobiol A, 134 (2000) 17.

15 K. Amano and T. Hinohara, J. Photochem. Photobiol A, 59 (1991) 43.

16 P. Bortolus and S. Monti, J. Phys Chem., 91 (1987) 5046. 
17 M. L. Bender and M. Komiyama, Cyclodextrin Chemistry, Springer, Berlin, 1978, Ch.2.

18 V. T. D'Souza and M. L. Bender, Acc. Chem. Res., 20 (1987) 146.

19 V. Ramamurthy and D. F. Eaton, Acc. Chem. Res., 21 (1988) 300.

20 V. Ramamurthy (ed.), Photochemistry in organized and constrained media, V. C. H. Publisher, New York, 1991.

2 I J. Szejtli, Chem. Rev., 98 (1998) 1743.

22 N. Chattopadhyay, J. Photochem. Photobiol A, 58 (1991) 31.

23 S. Kundu and N. Chattopadhyay, I. Molec. Struc., 344 (1995) 151.

24 G. S. Cox and N. J. Turro, J. Am. Chem. Soc., 106 (1984) 422.

25 M. P. Eastman, B. Freida, C. C. Hsu and C. A. Cheng, J. Phys. Chem. 92 (1988) 1682 .

26 N. Chattopadhyay, A. Samanta, T. Kundu and M. Chowdhury, J. Photochem. Photobiol. A, 48 (1989) 61 .

27 A. Samanta, N. Chattopadhyay, D. Nath, T. Kundu and M. Chowdhury, Chem. Phys. Letts., 121 (1985) 507.

28 T. A. Engeland, T. Bultmann, N. P. Earnsting, M. A. Rodriguez and W. Thiel. Chem. Phys, 163 (1992) 43.

29 J. Catalan, F. Fabero, M. S. Guijarro, R. M. Claramunt, M. D. Santa Maria, M. C. Foces-Foces, F. H. Cano. J. Elguero and R. Sastre, J. Am. Chem. Soc, 112 (1990) 747.

30 P. Purkayastha, P. K. Bhattacharyya, S. C. Bera and N. Chattopadhyay, Phys, Chem. Chem. Phys., 1 (1999) 3253.

31 P. Purkayastha and N. Chattopadhyay, Phys. Chem. Chem. Phys, 2 (2000) 203.

32 A. Onkelinx, F. C. De Schryver, L. Viaene, M. Van der Auweraer, K. Iwai, M. Yamamoto, M. Ichikawa, H. Masuhara, M. Maus and W. Rettig, J. Am. Chem. Soc., $118(1996) 1457$.

33 S. Kundu, S. C. Bera and N. Chattopadhyay, Ind. J. Chem. A, 37 (1998) 102.

34 K. K. Rohatgi-Mukherjec, Fundamentals of Photochemistry, Wiley Eastern, New Delhi, 1988 (Section 5.3).

35 S. Li and W. C. Purdy, Chem. Rev, 92 (1992) 1457. 